\title{
DISEÑO DE SISTEMAS DE ENERGÍA SOLAR FOTOVOLTAICA- APLICACIÓN EN EL PERÚ
}

\section{Teresa Núñez Zúñiga / Victor Manuel Cruz Ornetta}

\section{Resumen}

Objetivos: Presentar los criterios de diseño de un sistema de energía solar fotovoltaica para aplicaciones domésticas y la aplicación de los mismos para las diferentes regiones naturales del Perú. Materiales y métodos: Se buscó la información sobre los conceptos básicos de los sistemas y los criterios de diseño en la literatura a nivel nacional e internacional. En cuanto a la insolación solar, se obtuvo información del Ministerio de Energía y Minas. Resultados: Se diseñaron y se calculó el costo aproximado de sistemas domiciliarios típicos para la Costa, Sierra y Selva. Conclusiones: Los sistemas resultantes del diseño son factibles de implementar de forma costo-efectiva y podrían ser aplicados para solucionar las necesidades de lugares rurales de dificil acceso.

Palabras Clave: energía solar, radiación solar, energía renovable, energía limpia.

\section{Abstract}

Objectives: To present the criteria to design a solar photovoltaic energy system for domestic application and their application or the different natural regions in Peru. Materials and methods: Se busco la información sobre los conceptos básicos de los sistemas y los criterios de diseño en la literatura a nivel nacional e internacional. En cuanto a la información sobre las horas pico de sol se obtuvo información del Ministerio de Energia y Minas. It was looked for information about system basic concepts and design criteria in national and international sources. In relation with sun insolation it was obtained information from the Ministry of Energy and Mines. Results: It was designed systems and approximately computed the cost of typical home photovoltaic systems for the coastal, mountain and forest regions of Peru. Conclusions: It is feasible to implement the photovoltaic solar energy systems designed in cost - effective way and they could be very useful to provide energy to remote rural areas difficult to access in Peru.

Key words: solar energy, solar irradiation, renewable energy, clean energy. 


\section{INTRODUCCIÓN}

La energía solar fotovoltaica es una energía renovable muy importante para la provisión de energía en áreas geográficas de difícil acceso y que no cuentan con energía eléctrica comercial. Debido a la disminución experimentada en los costos de los sistemas de energía fotovoltaicos en los últimos años, estos sistemas se han hecho más accesibles y son una alternativa de solución para muchos lugares con carencias de energía. En ese sentido, el objetivo del presente estudio es proporcionar una metodología de diseño de los sistemas y aplicar esta metodología para diseñar sistemas típicos para la Costa, Sierra y Selva del Perú.

\section{MATERIALES Y MÉTODOS}

Para cumplir con el objetivo planteado en este estudio se realizo la revisión de diferentes documentos publicados en el ámbito internacional y a nivel nacional.

\section{RESULTADOS}

COMPONENTES Y CARACTERÍSTICAS DE LOS SISTEMAS DE ENERGÍA SOLAR FOTOVOLTAICA

El sistema de energía solar fotovoltaico básicamente está conformado por un panel de módulos fotovoltaicos, un banco de baterías $y$ una unidad de control [1-3]. El banco de baterías es necesario, ya que las características de la carga generalmente no coinciden con las características de la generación del panel fotovoltaico. En la figura 1 se muestra de manera esquemática el sistema fotovoltaico básico. Los parámetros básicos del subsistema son:

- Potencia de salida: Básicamente está determinado por la potencia de salida de los paneles.

- Autonomía: Es la cantidad de días que el sistema puede operar, satisfaciendo las necesidades de la carga, sin que exista un nivel mesurable de insolación.

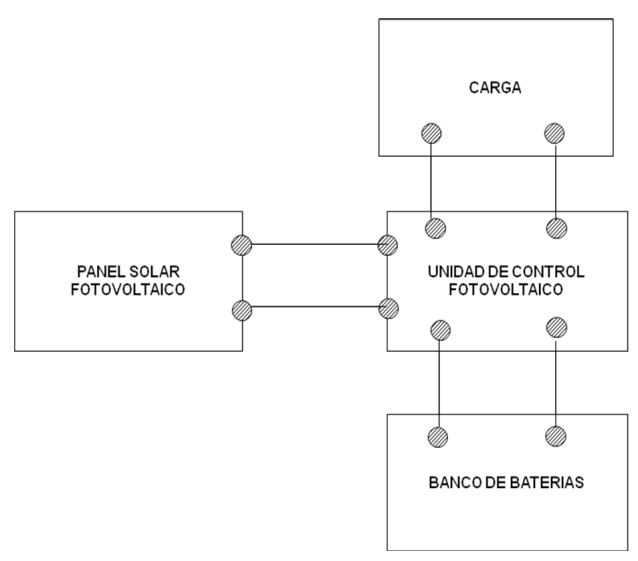

Fig. 1 El sistema solar fotovoltaico

\section{Módulos Fotovoltaicos}

En base a los módulos se conforman los paneles fotovoltaicos y, a su vez, en base a los paneles se forman los arreglos fotovoltaicos.

Los parámetros básicos de un módulo fotovoltaico y, por lo tanto, de un panel o un arreglo fotovoltaico, son: Potencia eléctrica, voltaje de trabajo e intensidad de corriente [4-8]

En la figura 2 se muestra la curva característica de la variación de 
corriente respecto de la tensión del módulo.

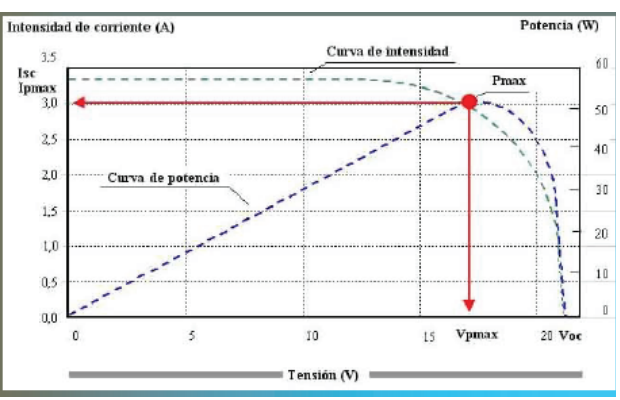

Fig. 2 Curva corriente vs voltaje de un módulo fotovoltaico

La potencia máxima de un módulo, panel o arreglo corresponde al máximo de irradiación solar $\left(1000 \mathrm{Wm}^{-2}\right)$. En el caso de un módulo se denota con el punto $\mathrm{P}_{\mathrm{M}}$. Permite calcular la energía diaria proporcionada por el panel, usando el número de horas de sol pico (h.s.p). La potencia disminuye de manera directamente proporcional con la irradiancia solar (ver figura 3).

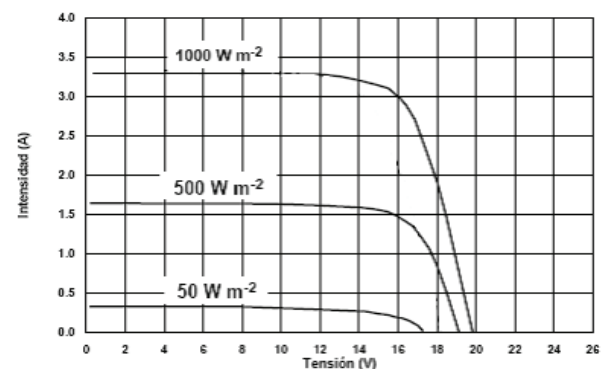

Fig. 3 Variación de la curva corriente vs voltaje de un módulo fotovoltaico en función de la irradiancia

El voltaje de trabajo es el que proporciona el panel o arreglo a la salida, oscilando entre 13 y 16 V. El valor depende de la intensidad de corriente proporcionada (a máxima corriente mínimo voltaje y viceversa: a minima corriente máximo voltaje. E1 voltaje se puede aumentar colocando más módulos o paneles en serie. El voltaje de circuito abierto del módulo disminuye con la temperatura, por lo que el rendimiento del panel disminuye con la temperatura (ver figura 4).

La intensidad de corriente depende del tamaño del panel o arreglo. La corriente se puede aumentar colocando más módulos o paneles en paralelo.

Las condiciones estándares de medición, son: irradiancia de 1000 $\mathrm{Wm}^{-2}$, espectro óptico de 1.5 , ángulo de incidencia de $0^{\circ} \mathrm{y}$ temperatura de operación de $25^{\circ} \mathrm{C}$.

Las condiciones nominales de operación, son: irradiancia de $800 \mathrm{Wm}^{-}$ ${ }^{2}$, espectro óptico de 1.5 , velocidad de viento de $1 \mathrm{~ms}^{-1}$, ángulo de incidencia. $0^{\circ}$, temperatura de operación: TONC, temperatura ambiente: $20^{\circ} \mathrm{C}$.

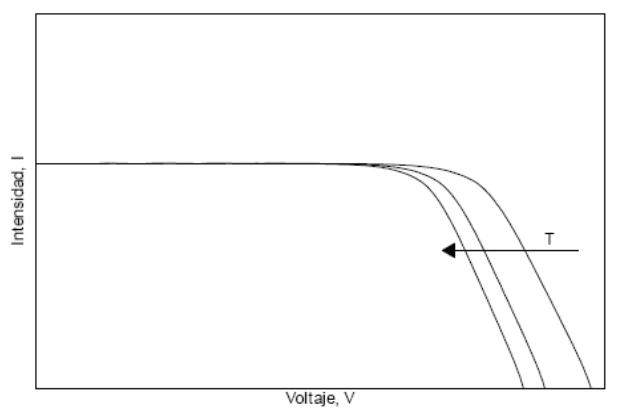

Fig. 4 Corriente vs voltaje de un módulo fotovoltaico en función de la temperatura

$\mathrm{Si}$ se sombrea parcialmente un panel, la presencia de células en sombra provoca diferencia de 
respuesta en la célula, pérdida de energía, calentamiento de la célula sombreada y posible destrucción de la célula.

La orientación óptima de los paneles fotovoltaicos [8] necesitaría que estuvieran en todo momento perpendiculares a la "línea imaginaria" que una el sol con la instalación fotovoltaica. Eso es lo que sucede en las instalaciones con seguidor de dos ejes, ya que en ese caso la instalación se mueve siguiendo el movimiento del sol a lo largo del día. Pero las instalaciones fijas deben orientarse para tratar de aprovechar al máximo la energía recibida del sol.

La instalación de los paneles fotovoltaicos está determinada por dos ángulos, el acimut "a" (ángulo que mide la desviación respecto al ecuador) y la inclinación o elevación " $\beta$ " (ángulo formado por la superficie del módulo y el plano horizontal). La posición idónea es con $\mathrm{a}=0^{\circ}$, es decir, en el caso del hemisferio sur se debe apuntar hacia el norte para que durante el día el panel capte la mayor cantidad de radiación posible. Es necesario tener cuidado para que no se produzcan sombras sobre los paneles o parte de ellos, especialmente hay que tomar en cuenta árboles, edificios, muros y las sombras que una fila de paneles puede producir sobre las otras. Una vez fijado el acimut, el parámetro que es determinante es la inclinación del panel, que se expresa como el ángulo beta (ß). Debido a que la máxima altura que alcanza el sol cada día varía según las estaciones, teniendo su máxi- mo en el día del solsticio de verano y su mínimo en el solsticio de invierno, lo ideal sería que el panel siguiese esta variación, pero esto no es posible por razones de coste. Se pueden dar a los paneles dos inclinaciones, una para los meses de verano y, otra, para los meses de invierno; aunque en ese caso también se complican las estructuras soporte, por lo que solo tiene sentido si hay un incremento considerable del consumo durante el verano. Normalmente se emplea una inclinación fija, la cual pueden ser diseñada de tal manera que se optimice la producción de energía eléctrica durante todo el año, máximizando la potencia media anual recibida, coincidiendo en la mayoría de los casos con la latitud del lugar de la instalación. En otros casos, se suele tomar un ángulo mayor, aproximadamente $15^{\circ}$, en beneficio de una mayor captación durante el invierno, cuando la insolación disminuye, a costa de una peor captación en verano, cuando hay una mayor cantidad de luz. En el caso que se desee optimizar la captación en el verano, la inclinación del panel sea menor que la latitud del lugar, aproximadamente en $15^{\circ}$. En cualquier caso, el ángulo de inclinación no debería ser menor a $15^{\circ}$ para que el módulo se pueda autolimpiar del polvo o de la lluvia.

\section{Baterias de Acumuladores}

La capacidad de la batería es la cantidad de carga en Ah que es capaz de alimentar en régimen de descarga, permaneciendo la variación de su f.e.m. dentro de límites especificados [1-3, 9,10]. 
Para cada tipo de batería su capacidad depende del régimen de descarga, esto es, dependiendo del valor de la corriente, de la temperatura y de la densidad del electrolito, en función del grado de ionización La capacidad .es proporcionada por el fabricante bajo la forma de tablas, con valores referidos a la temperatura de $25^{\circ} \mathrm{C}$ y normalizados para cada régimen de descarga. La capacidad de la batería se expresa en Ah y es denominada capacidad nominal, es decir, la máxima cantidad de carga que puede ser extraída del acumulador. Generalmente, para cada tipo de acumulador, el fabricante provee la capacidad nominal y las diversas corrientes de régimen de descarga para un valor de tensión, con los respectivos tiempos de descarga, estableciéndose un tiempo minimo de descarga para evitar que el acumulador sufra averías por corrientes elevadas, que el fabricante estipula.

El tiempo de descarga representa el periodo dado por el fabricante para que la batería alcance una tensión final de descarga, en un determinado régimen de descarga de corriente.

En sistemas solares, las baterías tienen que dar la energía sobre un tiempo considerablemente largo, y frecuentemente se descargan a niveles más bajos que en el caso de las baterias de arranque de los vehículos. Estas baterias de tipo ciclo profundo tienen capas de plomo más gruesas, que además brindan la ventaja de significativamente prolongar su vida, siendo relativamente grandes y pesadas por el plomo. Son compuestas de celdas de 2 voltios nominales que se juntan en serie para lograr baterías de 6, 12 o más voltios.

Las baterías liquidas de ciclo profundo (incluso las 'selladas'), son capaces de 400 ciclos. Las baterias de AGM y de Gel superan fácilmente 800 ciclos. Hay baterías de Gel para el uso industrial (por ejemplo los tipos OPzS o $\mathrm{OPzV}$ ), que pueden manejar bajo ciertas condiciones más de ¡10,000 ciclos! Los años de vida dependen entonces de su uso: si se conoce la profundidad de descarga y la cantidad de ciclos, se puede estimar su vida en años.

Todas las baterias de plomo-ácido necesitan una buena carga. Baterías de calidad, siempre llenas, sin sobrecargarlas, pueden vivir 20 años o más. $\mathrm{Si}$ se descargan frecuentemente en forma profunda tienen una vida más corta, y mantenerlos sin carga sobre un tiempo prolongado es su fin

En la práctica, esto significa instalar suficiente capacidad para descargar las baterías como máximo al 50\% de su capacidad nominal. Para ello, se debe dimensionar el panel con suficiente capacidad para cargarlas completamente. Tener suficiente capacidad instalada, además, tiene el importante benefició de aumentar las reservas, por ejemplo para los días con poco sol y para situaciones de emergencias cuando de repente se necesita más luz. Entonces, dependiendo de la necesidad de electricidad, es importante calcular un balance óptimo para la capacidad instalada. 
La temperatura tiene gran influencia sobre la batería. La temperatura entre 20 y $25^{\circ} \mathrm{C}$ es la óptima para una batería en uso. A más alta temperatura, la vida es más corta. Una temperatura de 10 grados arriba del óptimo puede cortar la vida por la mitad. Por otro lado, hay que tomar en cuenta que la capacidad de almacenar energía disminuye en temperaturas bajas. Entonces, para una batería sin uso, es preferible mantenerla a una temperatura más baja.

La temperatura también influye en cómo cargar una batería. Con el aumento de la temperatura, hay que disminuir el voltaje para evitar una gasificación, pero todavía asegurando una carga por completo. Recordamos que una gasificación en baterías selladas (libre de mantenimiento) no es recuperable: el líquido se pierde por las válvulas sin tener la posibilidad de rellenarlas. Por eso, todos los controladores buenos tienen una compensación de temperatura incorporada.

La eficiencia de las baterias varian según el tipo, la temperatura, la vejez, el estado de descarga y su calidad de construcción. Una batería descargada con una corriente alta en poco tiempo tiene menos capacidad que la misma descargada con una corriente pequeña sobre un tiempo prolongado. Normalmente se indican la capacidad de la batería descargada sobre 24 horas (a $25^{\circ} \mathrm{C}$ ), pero algunos fabricantes miden la capacidad hasta 100 horas y así indican un valor comparativo más alto de la competencia.
La eficiencia de la batería es la diferencia entre la cantidad de energia que entra en la bateria (cargando) y la que es disponible en la batería (descargando). Esta eficiencia de Coulomb (también llamada eficiencia de Faraday) en baterias de plomo normales es entre 70 y $85 \%$. Significa, por ejemplo, que de 100Ah producidos para cargar la batería, solamente entre 70 y 85Ah están disponible, el resto de la energía se pierde principalmente en calor. Las baterías de buena calidad, sobre todo los de tipo AGM, pueden tener una eficiencia de hasta $95 \%$.

\section{La unidad de control}

La unidad de control es necesaria para prolongar la vida útil de la batería. La función básica de un controlador es evitar la sobrecarga, de la batería. Si se permite que la batería sea sobrecargada rutinariamente se reducirá su esperanza de vida dramáticamente. Un controlador sensará el voltaje de la batería y reducirá o parará la carga de corriente cuando el voltaje llega a un voltaje suficientemente alto, lo cual es especialmente importante cuando se trata de baterías selladas, donde no se puede reemplazar el agua perdida durante la sobrecarga. En este caso, los controladores fotovoltaicos pueden abrir el circuito cuando las baterías están lo suficientemente cargadas sin ningún daño para los módulos. La mayoria de controladores fotovoltaicos simplemente abren o restringen el circuito entre la batería y el arreglo fotovoltaico, cuando el voltaje alcanza un punto precitado. Luego, cuando la batería absorbe el exceso de carga y el 
voltaje comienza a caer el controlador reconecta al arreglo [1-3,9]..

Algunos controladores tienen los umbrales de voltaje pre-fijados en fabrica y, en otros casos, pueden ser ajustados por el operador.

Las unidades de control son especificadas por su capacidad de corriente. En general, los controladores deberian ser capaces de soportar una sobre corriente de $25 \%$ de la capacidad nominal. Ello permite que el controlador pueda resistir cambios bruscos de insolación cuando la luz solar se incrementa, de lo contrario podrian dañarse.

Utilizar un controlador con una capacidad mayor que la generada permitirá una futura expansión de la carga $\mathrm{y}$, usualmente, no implica un costo adicional muy grande.

Un controlador también previene contra el flujo de corriente inversa en la noche, que es una pequeña cantidad de corriente que fluye de retorno al panel en la noche, descargando la bateria, aunque la perdida de potencia es no significativa.

Para poder cumplir con este objetivo, la unidad de control fotovoltaica deberá tener entre otras características: Voltajes regulados por compensación de temperatura, protección contra inducción electrostática, diodo de protección por bloqueo, protección contra inversión de polaridad, protección contra sobrecarga.

Dimensionamiento de un sistema solar fotovoltaico

Los criterios básicos para el dimensionamiento del sistemas solar foto- voltaico se presentan a continuación [1-3, 5-11].

Determinación de la potencia consumida

- Cálculo del total de los vatios -hora por día, considerando todos los electrodomésticos o equipos a ser utilizados.

- Cálculo del tamaño del panel es decir el total vatios-hora por día a ser generados por el panel fotovoltaico: Multiplicar el total de vatios-hora por día por 1.3. (para considerar las pérdidas en el sistema), para obtener los vatios- hora por día que deben ser proporcionados por el panel fotovoltaico.

Dimensionamiento de los módulos fotovoltaicos

- La potencia del panel se obtiene a partir del total de los vatios horadía a ser generados por el panel fotovoltaico dividido entre el número de horas pico de sol del peor mes, o entre el número de horas pico de sol promedio en el año.

- La corriente del panel se obtiene dividiendo la potencia del panel entre el voltaje del panel.

- El número de módulos en serie se obtiene dividiendo el voltaje del panel entre el de voltaje de los módulos.

- El número de módulos en paralelo se calcula dividiendo la corriente del panel entre la corriente del módulo.

- El número total de módulos resulta multiplicando el número de módulos en serie por le número de módulos en paralelo. 
Dimensionamiento del Banco de Baterias

- El tamaño del banco de baterías deberá cumplir con la autonomía y con la descarga diaria

a) Para considerar los días de autonomía se divide el total de vatios-hora por día por 0.85 (considerando las pérdidas de la batería) para obtener los vatios hora por día que deben ser proporcionados por la batería; luego se debe dividir el resultado entre 0.6 para considerar la profundidad de descarga. Finalmente, multiplicar el resultado por el número de días de autonomía.

b) Para considerar la descarga diaria máxima, dividir el total de vatios -hora-día por 0.85 (considerando las pérdidas en la batería) para obtener los vatios hora-día que deben ser proporcionados por la batería y luego dividir el resultado encontrado entre 0.15 para considerar la profundidad de descarga diaria.

- La Capacidad del Banco será la mayor de las capacidades de banco encontradas.
- El número de baterías en serie se obtiene dividiendo el voltaje del banco de baterías entre el de voltaje de la batería.

- El número de baterías en paralelo se obtiene dividiendo la Capacidad del Banco entre la capacidad de batería.

- El número total de baterías resulta de multiplicar el número de baterías en serie por el número de baterías en paralelo.

\section{Dimensionamiento de la Unidad de} Control

De acuerdo a la práctica estándar, el dimensionamiento del controlador de carga solar consiste en multiplicar la corriente de cortocircuito (Isc) del panel fotovoltaico y mutliplicarla por 1.3

\section{Hoja de cálculo para el dimensiona- miento de sistemas fotovoltaicos}

En el Anexo 1 se puede ver la hoja de cálculo utilizada para dimensionar los sistemas fotovoltaicos para la Costa, Sierra y Selva del Perú, y en la Tabla 1 se presentan los resultados de la aplicación de la hoja de cálculo. 
Teresa Núñez Zúñiga / Víctor Manuel Cruz Ornetta

Tabla 1 Dimensiones básicas de los sistemas solares para ciudades típicas de la Costa, Sierra y Selva del Perú

\begin{tabular}{|l|r|r|r|r|r|r|r|r|r|r|}
\hline & \multicolumn{3}{|c|}{ Sistema Fotovoltaico } & \multicolumn{3}{c|}{ Panel Fotovoltaico } & \multicolumn{2}{c|}{$\begin{array}{c}\text { Banco de bate- } \\
\text { rias }\end{array}$} & \multicolumn{2}{|c|}{$\begin{array}{c}\text { Unidad de con- } \\
\text { trol }\end{array}$} \\
\hline Lugar & $\begin{array}{c}\text { Energía } \\
\text { (Wh- } \\
\text { dia }^{-1} \text { ) }\end{array}$ & $\begin{array}{c}\text { Vol- } \\
\text { taje } \\
\text { (V) }\end{array}$ & $\begin{array}{c}\text { Auton } \\
\text { (dias) }\end{array}$ & $\begin{array}{c}\text { Co- } \\
\text { rriente } \\
\text { (A) }\end{array}$ & $\begin{array}{c}\text { Vol- } \\
\text { taje } \\
\text { (V) }\end{array}$ & $\begin{array}{c}\text { In- } \\
\text { clin. } \\
\left({ }^{\circ}\right)\end{array}$ & $\begin{array}{c}\text { Capaci- } \\
\text { dad (Ah) }\end{array}$ & $\begin{array}{c}\text { Vol- } \\
\text { taje } \\
\text { (V) }\end{array}$ & $\begin{array}{c}\text { Capaci- } \\
\text { dad (A) }\end{array}$ & $\begin{array}{c}\text { Volta- } \\
\text { je (V) }\end{array}$ \\
\hline Cuzco & 1092 & 12 & 5 & 26.3 & 12 & 29 & 892.2 & 12 & 40.6 & 12 \\
\hline Ica & 1092 & 12 & 5 & 23.7 & 12 & 28 & 892.2 & 12 & 33.8 & 12 \\
\hline Pucallpa & 1092 & 12 & 5 & 39.4 & 12 & 23 & 892.2 & 12 & 60.8 & 12 \\
\hline
\end{tabular}

\section{CONCLUSIONES Y RECOMENDACIONES}

Se ha cumplido con los objetivos del estudio, ya que se han diseñado los sistemas de energía solar fotovol- taicos para las tres regiones naturales del Perú. Se recomienda verificar de manera experimental la metodología del dimensionamiento de dichos sistemas para validarla. 


\section{REFERENCIAS BIBLIOGRÁFICAS}

1. PERPIÑÁN O, CASTRO M, COLMENAR A. Diseño de sistemas fotovoltaicos Madrid: Promotora General de Estudios S.A.; 2012.

2. MORENO N, GRACIA L. Instalaciones de energía fotovoltaica 1 ra Edicion y 3ra Impresión. Madrid: Ibergarceta Publicaciones S.L.;2010

3. MÉNDEZ J, CUERVO R. Energía Solar Fotovoltaica. $4^{\text {ta }}$ Edición. Madrid: Fundación Confemetal; 2009

4. SOLARWORLD [Internet]. Hillsboro: SolarWorld Americas; [ citado 30 oct 2013]. Sunmodule SW 85 poly R5A [aprox. 2 pantallas]. Disponible en: http:// www.solarworld-usa.com/ /media/www/files/datasheets/sunmodule-offgrid/sunmodule-off-grid-solar-panel-85-poly-r5a.pdf

5. GASPARIN F. Desenvolvimento de um tracador de curvas caracteristicas de Módulos Fotovoltaico [tesis de maestria]. Porto Alegre RS: UFRGS; 2009.

6. PRIEB C. Desenvolvimento de um Sistema de Ensaio de Módulos Fotovoltaicos [tesis de maestria]. Porto Alegre RS: UFRGS; 2002.

7. ALMEIDA L. Analise e modelagem geométrica da potência gerada por um sistema híbrido solar fotovoltaico eólico [tesis de doctorado]. Sao Paulo: Universidade Estadual Paulista «Júlio de Mesquita Filho»; 2007

8. BÜHLER A. Estudo de Técnicas de Determinação Experimental e PósProcessamento de Curvas Características de Módulos Fotovoltaicos [tesis de doctorado]. UFRGS, Porto Alegre RS, 2011.

9. SANDIA NATIONAL LABORATORIES. Stand- alone Photovoltaic Systems - A Handbook of Recommended Design Practices. Springfield: National Technical Information Service U.S. Deparment of Commerce; 1995.

10. CENTRAL ELECTROCHEMICAL RESEARCH INSTITUTE (CSIR), Solar Energy Centre (MNRE). Design and Development of Batteries for Solar Photovoltaic Applications [Internet]. Nueva Delhi: CSRI y MNRE; 2012 [citado el 30 de oct 2013. Disponible en http:/ / mnre.gov.in/file-manager/UserFiles/report_ batteries_solar_photovoltaic_applications.pdf

11. SERVICIO NACIONAL DE METEOROLOGÍA E HIDROLOGÍA. Atlas de energía solar del Perú. [Internet]. Lima: Servicio Nacional de Meteorología e Hidrología; 2003 [citado 2012 Jun 26]. Disponible en: http://dger.minem. gob.pe/atlassolar/ATLAS_SOLAR.pdf 
ANEXO 1

\begin{tabular}{|c|c|c|c|c|c|c|c|c|}
\hline \multicolumn{9}{|c|}{ CARACTERÍSTICAS DE LA CARGA } \\
\hline & & \multicolumn{2}{|c|}{ voltaje } & \multicolumn{2}{|c|}{$\begin{array}{l}\text { poten- } \\
\text { cia }\end{array}$} & $\begin{array}{c}\text { horas } \\
\text { /dia }\end{array}$ & vatios-hora/dia & \multirow[b]{2}{*}{ Whdia $^{-1}$} \\
\hline 1 & lampara flourescente & 12 & $\mathrm{~V}$ & 18 & $\mathrm{~W}$ & \begin{tabular}{l|l|}
4 & $\mathrm{~h}$ \\
\end{tabular} & 72 & \\
\hline 1 & ventilador & 12 & $\mathrm{~V}$ & 60 & $\mathrm{~W}$ & \begin{tabular}{l|l|}
2 & $\mathrm{~h}$ \\
\end{tabular} & 120 & Whdia $^{-1}$ \\
\hline \multirow[t]{2}{*}{1} & refrigerador & 12 & $\mathrm{~V}$ & 75 & $\mathrm{~W}$ & \begin{tabular}{l|l|}
12 & $\mathrm{~h}$ \\
\end{tabular} & 900 & Whdía $^{-1}$ \\
\hline & Energía total & 12 & $\mathrm{~V}$ & & & & 1092 & Whdia $^{-1}$ \\
\hline \multicolumn{9}{|c|}{ CARACTERÍSTICAS CLIMÁTICAS Y UBICACIÓN } \\
\hline \multicolumn{7}{|c|}{ Lugar } & \multicolumn{2}{|l|}{ Ica } \\
\hline \multicolumn{7}{|c|}{ Horas pico de sol } & \multicolumn{2}{|c|}{\begin{tabular}{l|l}
5 & $\mathrm{~h}$
\end{tabular}} \\
\hline \multicolumn{7}{|c|}{ Latitud } & 13 & $\circ$ \\
\hline \multicolumn{9}{|c|}{ CARACTERÍSTICAS DEL SISTEMA } \\
\hline \multicolumn{7}{|c|}{ Energía total a alimentar } & 1092 & Whdia $^{-1}$ \\
\hline \multicolumn{7}{|c|}{ Voltaje } & 12 & V \\
\hline \multicolumn{7}{|c|}{ Autonomía } & 5 & dias \\
\hline \multicolumn{9}{|c|}{ ESPECIFICACIONES DEL PANEL FOTOVOLTAICO } \\
\hline \multicolumn{7}{|c|}{ Energía del panel } & 1419.6 & Whdia $^{-1}$ \\
\hline \multicolumn{7}{|c|}{ Potencia del panel } & 283.9 & W \\
\hline \multicolumn{7}{|c|}{ Voltaje del panel } & 12 & V \\
\hline \multicolumn{7}{|c|}{ Corriente del panel } & 23.7 & A \\
\hline \multicolumn{7}{|c|}{$\begin{array}{l}\text { Inclinación del panel optimizada para captación en } \\
\text { invierno }\end{array}$} & 28 & $\circ$ \\
\hline \multicolumn{7}{|c|}{ Voltaje del módulo } & 12 & V \\
\hline \multicolumn{7}{|c|}{ Corriente en cortocircuito del módulo } & 5.2 & A \\
\hline & orriente nominal del mć & & & & & & 4.8 & A \\
\hline & ódulos en serie & & & & & & 1 & \\
\hline & ódulos en paralelo & & & & & & 5 & \\
\hline & tal de módulos & & & & & & 5 & \\
\hline & ESP & IFICA & $\mathrm{ICIO}$ & DNES & DEI & BANCC & O DE BATERÍAS & \\
\hline & $\begin{array}{l}\text { apacidad del banco pa } \\
\text { aria }\end{array}$ & profu & ndic & dad d & de de & scarga & 713.73 & Ah \\
\hline & apacidad del banco par & utono & mía & & & & 892.16 & Ah \\
\hline & apacidad del banco & & & & & & 892.16 & Ah \\
\hline & bltaje del banco & & & & & & 12 & V \\
\hline & apacidad de la batería & & & & & & 99.50 & Ah \\
\hline & bltaje de la batería & & & & & & 12 & V \\
\hline & aterías en paralelo & & & & & & 8 & \\
\hline & aterias en serie & & & & & & 1 & \\
\hline & tal de baterias & & & & & & 8 & \\
\hline & ESPE & FICAC & CION & NES I & DE L & A UNID & AD DE CONTROL & \\
\hline & tencia de la unidad de & atrol & & & & & 405.6 & W \\
\hline & apacidad de la unidad d & ontro & & & & & 33.8 & $\mathrm{~A}$ \\
\hline & ltaje de la unidad de cc & & & & & & 12 & $\mathrm{~V}$ \\
\hline
\end{tabular}

